\title{
Electrospun Dual-Layer Mats with Covalently Bonded ZnO Nanoparticles for Moisture Wicking and Antibacterial Textiles
}

\author{
Yuliang Dong, ${ }^{1,2,3}$ Noreen L. Thomas, ${ }^{3,} *$ Xuehong $\mathrm{Lu}^{1,2, *}$ \\ ${ }^{1}$ School of Materials Science and Engineering, Nanyang Technological University, 50 Nanyang \\ Avenue, Singapore 639798 \\ ${ }^{2}$ Institute for Sport Research, Nanyang Technological University, 50 Nanyang Avenue, Singapore \\ 639798 \\ ${ }^{3}$ Department of materials, Loughborough University, Loughborough, Leicestershire, LE11 3TU, \\ United Kingdom
}

\begin{abstract}
The fast growing demand for advanced sportswear suitable for tropical countries has led to research on fabrics with both moisture wicking and anti-bacterial properties. In this work, to improve the water transport behaviour, dual-layer nanofibrous nonwoven mats composed of a hydrophilic polyacrylonitrile (PAN) outer layer and a hydrophobic poly(vinylidene fluoride) (PVDF) inner layer are fabricated by electrospinning. The distinct difference in surface hydrophobicity between the inner and outer layers induces a push-pull effect to transport water from the inner to the outer surface efficiently. To render an antibacterial property, zinc oxide ( $\mathrm{ZnO}$ ) nanoparticles are covalently attached to the PVDF nanofibers. The good anti-wash properties and anti-bacterial function of the ZnO-PDVF/PAN dual layer mats are demonstrated.
\end{abstract}

\section{Keywords}

Electrospinning, textile, moisture transport, zinc oxide, antibacterial

\section{Introduction}

In the field of sportswear, moisture management and antibacterial properties of the textiles are two critical issues for comfort and well-being, especially for people living in tropical countries. Since both the temperature and humidity in these countries are fairly high, people may suffer excessive perspiration in sports activities, and the hot and humid environment is also a favourable condition for microbial growth. ${ }^{1}$ 
In tropical environments, the surface of textiles will dry slowly due to the high humidity, hindering moisture transport. Thus the textiles used need to possess good moisture management properties to maintain the thermal balance of the wearer. ${ }^{2-4}$ If excessive sweat cannot be transported away fast enough, the accumulated sweat on the skin will also give rise to an uncomfortable feeling of dampness. In addition, contact between the skin and the wet inner surface of textiles will cause an increase in friction between the skin and textile, which could affect wear comfort or even cause skin irritation. ${ }^{5-9}$ In textiles, the micro-grooves on the fiber surface foster capillary absorbency, siphoning moisture, which then diffuses and spreads over the fiber surface to facilitate dissipation. To improve moisture transport properties of textiles, various approaches have been adopted. For example, hydrophilic surface modification of hydrophobic textile fibers ${ }^{10,11}$ can provide moisturewicking fabrics through enhancing surface moisture transport, while retaining the good water release property of the hydrophobic fibers. Reduction in textile fibre diameter can help to increase the water evaporation rate and boost the capillary effect of the textiles. ${ }^{12}$ The use of dual-layer textiles composed of a hydrophobic inner layer and a hydrophilic outer layer is also an effective strategy to transport moisture through the textiles. In such dual-layer textiles, which are also called 'push-pull' fabrics, the sweat on the skin can diffuse into the inner layer and be 'pushed' from the hydrophobic inner layer and 'pulled' into to the hydrophilic outer layer because of the large difference in hydrophobicity between the two layers. ${ }^{3,13}$ The hydrophobic inner layer, consisting of fibers of non-absorbent materials, does not absorb the sweat although there are plenty of surface grooves and spaces between the fibers to hold a small amount of sweat. The absorbent material in the outer layer draws the sweat away from the inner layer via hydrophilic force and capillary action, while the non-absorbent inner layer keeps the skin dry. Based on this mechanism, the hydrophobic inner layer should be fairly thin in order to let the sweat pass through it quickly. Recently, using electrospun nanofibrous mats as model systems, it was demonstrated that a synergistic combination of these approaches could further improve moisture transport properties of dual-layer textiles. ${ }^{14}$

A warm, damp textile next to the skin provides a good substrate for the growth of micro-organisms. Although a broad range of micro-organisms can coexist in natural equilibrium with the human body, rapid and uncontrolled growth of microbes can lead to some serious problems, including unpleasant odour, the increase of potential health risks and degradation of the fibers. ${ }^{15-17}$ Since conventional fabrics do not have antibacterial properties, a variety of antibacterial agents have been loaded into fabrics, or attached directly onto the surface of the fabrics to render textiles antibacterial. For example, by simultaneous sonochemical dyeing and coating, antibacterial metal oxide nanoparticles have been coated onto fabrics. ${ }^{18}$ To achieve antibacterial properties, polylactide/zinc oxide 
nanocomposite filaments have been fabricated by a melt-spinning process. ${ }^{19}$ Zinc oxide $(\mathrm{ZnO})$ is widely used as an antibacterial agent because it is nontoxic, biosafe and has low cost. ${ }^{20-25}$

It is also worth noting that moisture transport and antibacterial properties are closely related. Textiles with good moisture management properties could provide a drier environment between skin and textiles, and hence lower the speed of bacterial growth. Thus novel design strategies are needed to address both moisture transport and antibacterial issues. In this work, for the first time, antibacterial properties are introduced into a push-pull dual-layer mat, which is composed of a thick hydrophilic polyacrylonitrile (PAN) nanofibrous layer and a thin hydrophobic polyvinylidene fluoride (PVDF) nanofibrous layer with covalently bonded $\mathrm{ZnO}$ nanoparticles on the PVDF nanofibers. PVDF was chosen as the inner layer of the dual-layer mats mainly for two reasons. Firstly, PVDF is hydrophobic, which ensures that the inner side of the textile will not stick to wet skin, while water can be pulled out by the hydrophilic outer layer (PAN). Secondly, the chemical structure of PVDF allows the $\mathrm{ZnO}$ nanoparticles to be covalently attached onto the functionalized PVDF. Compared with the approach of loading nanoparticles into the fibers, this approach can increase the direct contact area of the antibacterial agent with the surrounding media, providing a more efficient antibacterial function. Also the covalent bonding between the nanoparticles and the nanofibers can prevent the detachment of the nanoparticles, leading to a more durable antibacterial property while also overcoming the potentially harmful effect of the nanoparticles in contact with skin. More importantly, this design can give the dual-layer mat both good moisture transport and antibacterial functions. In this paper, the unique structure and morphology of the hybrid nanofibrous mats are reported, and the resultant good antibacterial, anti-wash, and moisture management properties of the mats are demonstrated.

\section{Experimental Work}

\subsection{Materials}

PAN(Mw $=150,000)$, Zinc nitrate hexahydrate $\left(\mathrm{Zn}\left(\mathrm{NO}_{3}\right)_{2} \cdot 6 \mathrm{H}_{2} \mathrm{O}, 98 \%\right)$, Hexamethylenetetramine (HMTA, 99\%), Zinc acetate dihydrate $\left(\mathrm{Zn}\left(\mathrm{CH}_{3} \mathrm{COO}\right)_{2} \cdot 2 \mathrm{H}_{2} \mathrm{O}, 98 \%\right)$, (3-Aminopropyl) trimethoxysilane (APTES, 98\%), sodium hydroxide (NaOH, 97\%), 1,3-diaminopropane (DAP, 99\%), acetone (99.5\%), and ethanol (99.5\%) were purchased from Aldrich Chemical Company Inc(USA). N, N-Dimethylformamide (DMF, 99\%) was purchased from Tedia Company Inc (USA). Poly(vinylidene fluoride-co-hexafluoropropene) (solef 11008, Mw=115,000) was purchased from 
Solvay Solexis Inc. (USA). It is referred to as PVDF in the following sections for simplicity. All materials were used without further purification.

\subsection{Preparation of cross-linked PVDF nanofibrous mats}

Cross-linked PVDF nanofibrous mats were prepared according to the procedure reported by Zhou et al. ${ }^{26}$ DAP was added to PVDF DMF/acetone solution (18 wt\% PVDF), followed by magnetic stirring for 1 hour. The solution was electrospun into nonwoven nanofibrous mats at an applied voltage of $18 \mathrm{kV}$ and feeding rate of $0.20 \mathrm{ml} / \mathrm{h}$. A grounded aluminium foil was located at a fixed distance of $15 \mathrm{~cm}$ from the needle tip to collect the nanofibrous mats. Electrospinning was carried out for 5 minutes for standard mats and for 3 hours for thick mats. Then the mats were cured in an oven at $120^{\circ} \mathrm{C}$ for 1 hour. The mats are denoted as PVDF and PVDF-thick, respectively.

\subsection{Preparation of ZnO-attached PVDF nanofibrous mats and dual-layer mats}

It has been reported that silane-functionalized PVDF nanofibers provide a versatile platform for covalently attaching inorganic oxide nanoparticles (such as $\mathrm{SiO}_{2}, \mathrm{TiO}_{2}$ and $\mathrm{Al}_{2} \mathrm{O}_{3}$ ) on PVDF nanofibers. ${ }^{25-28}$ In this work, the reported procedure was modified for covalently attaching $\mathrm{ZnO}$. In the first step, PVDF and PVDF-thick were immersed in $10 \mathrm{wt} \%$ APTES ethanol solution for 10 hours at $70{ }^{\circ} \mathrm{C}$, to allow the silane group to be grafted on to the surface of the fibers. The resultant nanofibrous mats are denoted as Si-PVDF and Si-PVDF-thick, respectively. In the second step, the Si-PVDF, PVDF and Si-PVDF-thick were immersed in $\mathrm{Zn}\left(\mathrm{CH}_{3} \mathrm{COO}\right)_{2} \cdot 2 \mathrm{H}_{2} \mathrm{O}$ and $\mathrm{NaOH}$ blended ethanol solution $\left(0.01 \mathrm{~mol} / \mathrm{L} \mathrm{Zn}\left(\mathrm{CH}_{3} \mathrm{COO}\right)_{2} \cdot 2 \mathrm{H}_{2} \mathrm{O}\right.$ and $\left.0.02 \mathrm{~mol} / \mathrm{L} \mathrm{NaOH}\right)$ for 30 minutes at $60^{\circ} \mathrm{C}$, then annealed at $120^{\circ} \mathrm{C}$ for 10 minutes. The samples obtained are denoted as seed-Si-PVDF, seedPVDF and seed-Si-PVDF-thick. In the third step, seed-Si-PVDF and seed-PVDF were immersed in $\mathrm{Zn}\left(\mathrm{NO}_{3}\right)_{2}$ and HMTA blend solution $\left(0.05 \mathrm{~mol} / \mathrm{L} \mathrm{Zn}\left(\mathrm{NO}_{3}\right)_{2} \cdot 6 \mathrm{H}_{2} \mathrm{O}\right.$ and $\left.0.05 \mathrm{~mol} / \mathrm{L} \mathrm{HTMA}\right)$ at $90^{\circ} \mathrm{C}$ for 1 hour, then rinsed and dried at $60^{\circ} \mathrm{C}$ for 2 hours. These products are denoted as ZnO-PVDF and ZnO-PVDF-no silane, respectively. Using the same route, seed-Si-PVDF-thick mats were immersed in $\mathrm{Zn}\left(\mathrm{NO}_{3}\right)_{2}$ and HMTA blend solutions for 0.5 hour, 1 hour and 3 hours, respectively, and then rinsed and dried at $60^{\circ} \mathrm{C}$ for 2 hours. They are denoted as 0.5-ZnO-PVDF-thick, 1-ZnOPVDF-thick and 3-ZnO-PVDF-thick, respectively.

PAN solution in DMF (7 wt\% PAN) was prepared by dissolving PAN powder in DMF under continuous magnetic stirring. The ZnO-PVDF nanofibrous mats obtained in the previous step were 
peeled off from the aluminum foil. Each of them had one surface with covalently attached $\mathrm{ZnO}$ nanoparticles and the other surface (which had been in touch with the aluminum foil) without $\mathrm{ZnO}$. PAN nanofibers were then electrospun onto the surfaces with and without $\mathrm{ZnO}$, respectively, for 1.5 hours using the setup mentioned above. The combined mats consisting of a PAN layer (around $100 \mu \mathrm{m}$ thick) and a ZnO-PVDF layer (around $5 \mu \mathrm{m}$ thick) were then laminated together using a paper laminator (PRO $\mathrm{BiO} 320$ ) at $70^{\circ} \mathrm{C}$ and at a rolling speed of $50 \mathrm{rpm}$. The resultant dual-layer mats are denoted as PVDF-ZnO/PAN (PAN electrospun onto the surface with $\mathrm{ZnO}$ ) and ZnOPVDF/PAN (PAN electrospun onto the surface without $\mathrm{ZnO}$ ), respectively. PAN nanofibers were also directly electrospun onto aluminum foil to make a reference sample, which is denoted as PAN.

\subsection{Characterization}

Sample morphologies were observed using a field-emission scanning electron microscopy (FESEM, JEOL 7600F). FESEM images were obtained under an accelerating voltage of $5 \mathrm{kV}$ and at a working distance of $8 \mathrm{~mm}$. All samples were coated with a thin layer of gold before imaging. XRD tests were conducted using a XRD-6000 Shimadzu diffractometer. Moisture transport ability of the samples was measured using a Moisture Management Tester (MMT). FTIR spectroscopy was recorded using a PerkinElmer (Frontier) spectrometer. Contact angles of the samples were measured using a contact angle tester (dataphysics, OCA 15 PRO)

To test the anti-wash property, the ZnO-PVDF and ZnO-PVDF-no silane samples were immersed in detergent aqueous solution $(4 \mathrm{~g} / \mathrm{L})$. The washing was conducted for 20 hours and 40 hours with continuous shaking. Subsequently, the mats were immersed in deionized water for two hours with continuous shaking, and then rinsed with deionized water several times to remove the detergent and other chemicals. The fibers after washing are denoted as washed-20h-ZnO-PVDF, washed-40hZnO-PVDF, washed-20h-ZnO-PVDF-no silane and washed-40h-ZnO-PVDF-no silane.

Bacterial cultures of E. coli-K12 (Wild Type) were prepared in 100ml LB Broth, Miller, inoculated and incubated at $37^{\circ} \mathrm{C}$ overnight. LB Agar plates were seeded with $1 \mathrm{ml}$ of overnight culture. Discs of test material were placed onto plates and incubated at $37{ }^{\circ} \mathrm{C}$ overnight. The inhibition zones were photographed and the samples were observed by FESEM. 


\section{Results and Discussion}

In this work, both PAN and PVDF nanofibers were prepared by electrospinning, while ZnO nanoparticles were covalently attached onto the surface of the electrospun PVDF nanofibers to form hybrid mats through a sol-gel-based process, as shown in Scheme 1. The PVDF nanofibrous mats were cross-linked in order to improve their structural integrity for facilitating the sol-gel reaction. The hybrid and PAN mats are firmly stacked together via a simple laminating process, and the laminated mats are denoted as ZnO-PVDF/PAN dual-layer mats. In order to illustrate the properties of the ZnO-PVDF/PAN dual-layer mats, some reference samples were also prepared. The sample preparation conditions are summarized in Table 1.

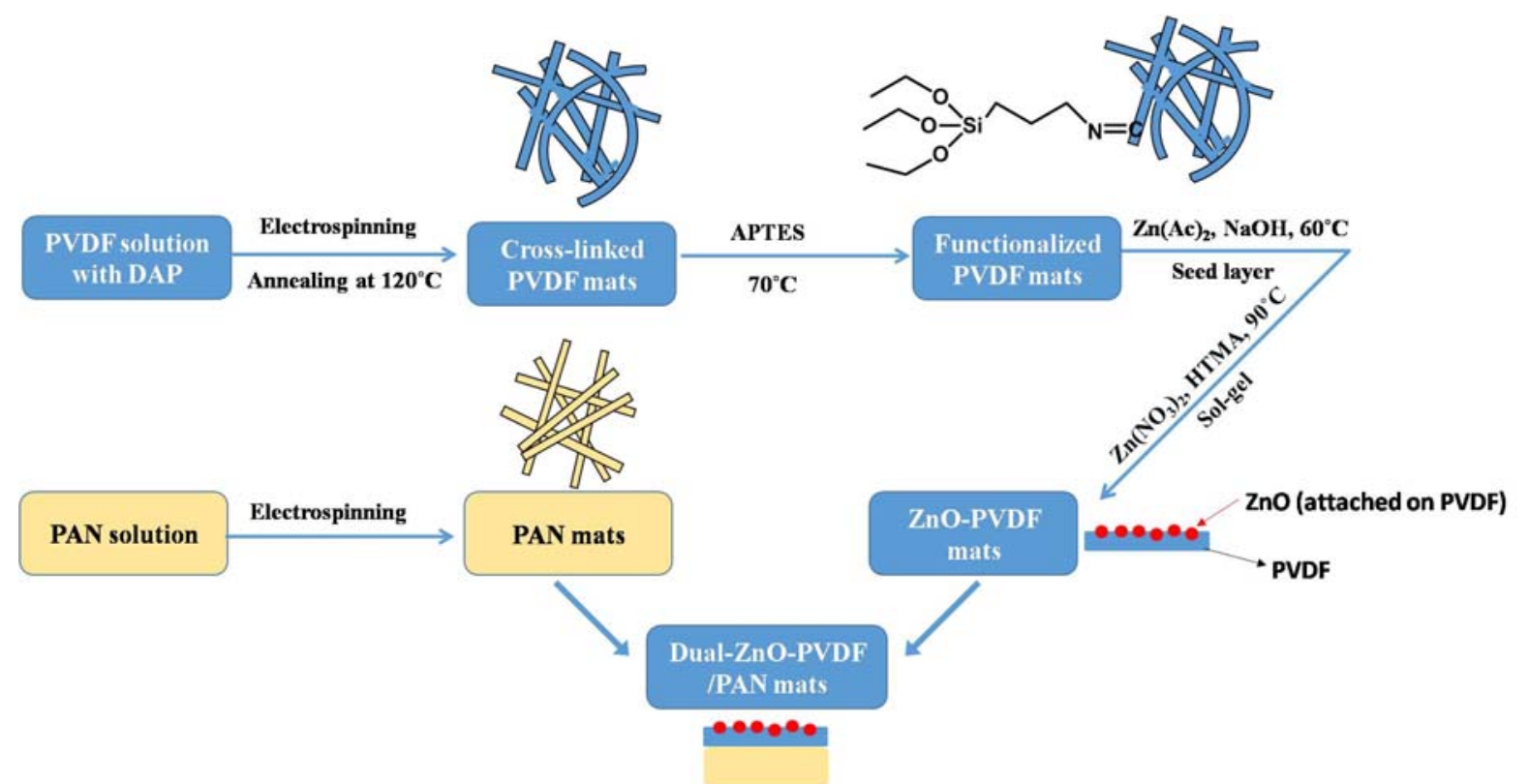

Scheme 1. Preparation of ZnO-PVDF/PAN dual-layer mats

Table 1. Sample preparation conditions

\begin{tabular}{|l|l|l|l|l|l|l|}
\hline Sample Name & Polymer & $\begin{array}{l}\text { Spinning } \\
\text { time }\end{array}$ & $\begin{array}{l}\text { Silane } \\
\text { attachment }\end{array}$ & Seeding & $\begin{array}{l}\text { Sol-gel } \\
\text { time }\end{array}$ & $\begin{array}{l}\text { Single/ } \\
\text { Dual-layer }\end{array}$ \\
\hline PVDF-thick & PVDF & $3 \mathrm{hr}$ & No & No & - & Single layer \\
\hline Si-PVDF-thick & PVDF & $3 \mathrm{hr}$ & Yes & No & - & Single layer \\
\hline seed-Si-PVDF-thick & PVDF & $3 \mathrm{hr}$ & Yes & Yes & - & Single layer \\
\hline 0.5-ZnO-PVDF-thick & PVDF & $3 \mathrm{hr}$ & Yes & Yes & $0.5 \mathrm{hr}$ & Single layer \\
\hline 1-ZnO-PVDF-thick & PVDF & $3 \mathrm{hr}$ & Yes & Yes & $1 \mathrm{hr}$ & Single layer \\
\hline 3-ZnO-PVDF-thick & PVDF & $3 \mathrm{hr}$ & Yes & Yes & $3 \mathrm{hr}$ & Single layer \\
\hline
\end{tabular}




\begin{tabular}{|l|l|l|l|l|l|l|}
\hline PVDF & PVDF & $5 \mathrm{~min}$ & No & No & - & Single layer \\
\hline ZnO-PVDF & PVDF & $5 \mathrm{~min}$ & Yes & Yes & $1 \mathrm{hr}$ & Single layer \\
\hline ZnO-PVDF-no silane & PVDF & $5 \mathrm{~min}$ & No & Yes & $1 \mathrm{hr}$ & Single layer \\
\hline PAN & PAN & $1.5 \mathrm{hr}$ & No & No & - & Single layer \\
\hline ZnO-PVDF/PAN & PVDF & $5 \mathrm{~m}+1.5 \mathrm{~h}$ & Yes & Yes & $1 \mathrm{hr}$ & Dual-layer \\
\hline PVDF-ZnO/PAN & PVDF & $5 \mathrm{~m}+1.5 \mathrm{~h}$ & Yes & Yes & $1 \mathrm{hr}$ & Dual-layer \\
\hline
\end{tabular}

\subsection{Structure and morphology of the PVDF nanofibrous mats with covalently attached ZnO}

To confirm the successful preparation of ZnO-PVDF hybrid mats, following the preparation route, the morphologies of the nanofibrous mats were observed step by step. Since a relatively large amount of $\mathrm{ZnO}$ is required for composition analysis by EDX, relatively thick mats (thickness $\approx 100$ $\mu \mathrm{m}$ ) were used for the SEM characterization. As shown in Figs. 1a-1c, the morphology of the mats does not show any obvious change after the attachment of silane, and the coating of the $\mathrm{ZnO}$ seed layer. The attachment of silane groups and the seeding layer were confirmed by FTIR (Fig. S1). After the sol-gel reaction, large amounts of $\mathrm{ZnO}$ nanoparticles are attached onto the nanofibers. With the increase of the sol-gel reaction time, the $\mathrm{ZnO}$ nanoparticles gradually grow into nanorods. The EDX results in Table 2 show the increases of both $\mathrm{Zn}$ and $\mathrm{O}$ contents with the reaction time, corroborating the SEM observation.

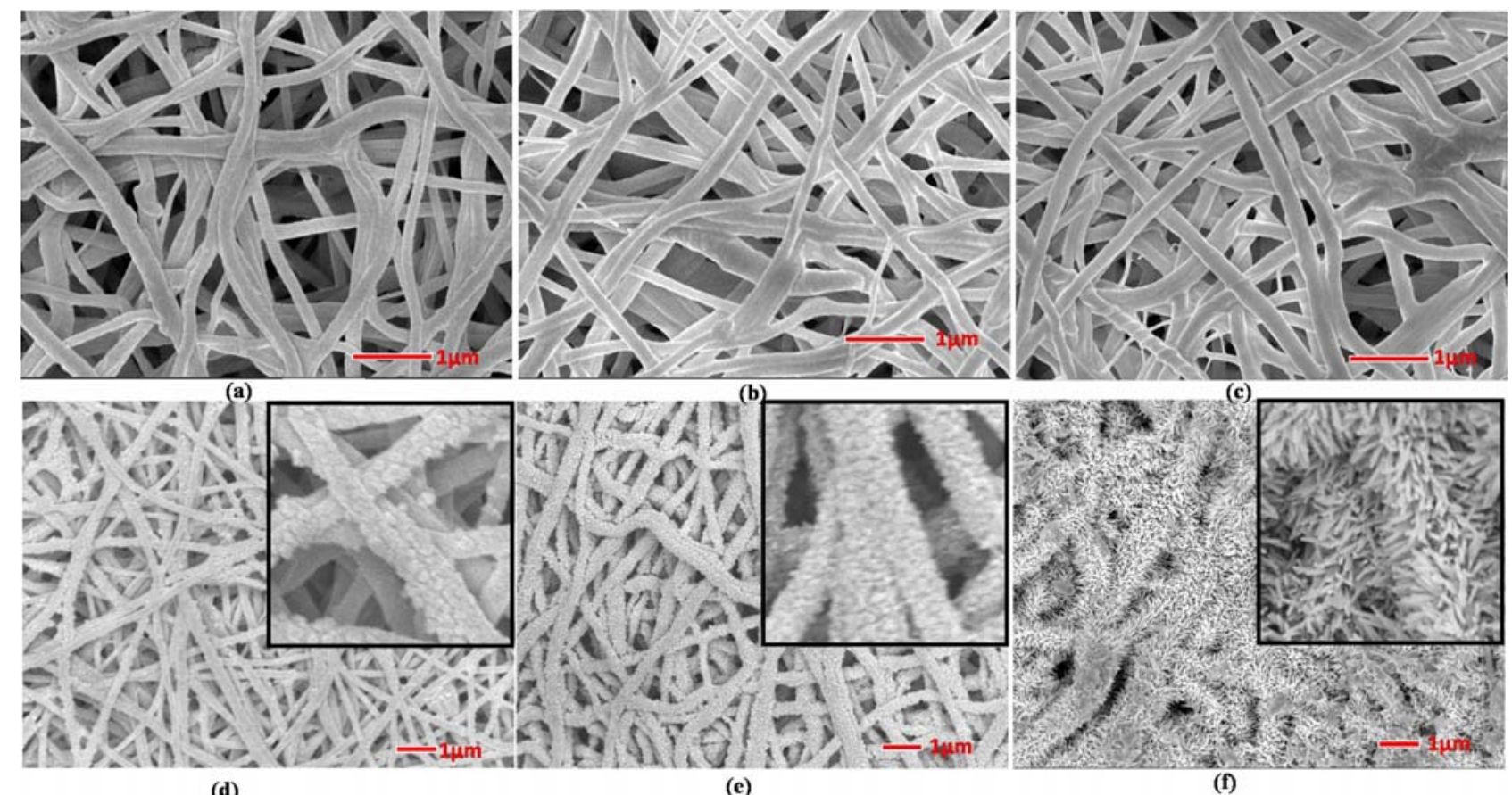


Figure.1 FESEM micrographs showing the morphologies of (a) PVDF-thick, (b) Si-PVDF-thick, (c) seed-Si-PVDF-thick, (d) 0.5-ZnO-PVDF-thick, (e) 1-ZnO-PVDF-thick and (f) 3-ZnO-PVDFthick mats.

Table 2. EDX results of 0.5-ZnO-PVDF-thick, 1-ZnO-PVDF-thick and 3-ZnO-PVDF-thick mats

\begin{tabular}{|c|c|c|c|}
\hline Samples & Zn Atomic (\%) & $\mathrm{Zn} / \mathrm{F}$ & $\mathrm{O} / \mathrm{F}$ \\
\hline 0.5-ZnO-PVDF-thick & 0.82 & 0.02 & 0.06 \\
\hline 1-ZnO-PVDF-thick & 1.94 & 0.04 & 0.06 \\
\hline 3-ZnO-PVDF-thick & 24.20 & 0.92 & 0.90 \\
\hline
\end{tabular}

Since the overgrowth of $\mathrm{ZnO}$ nanorods may block pores in the hybrid mats, hindering moisture wicking in the mats, in the following experiments, the time for the sol-gel reaction was fixed at 1 hour. After 1-hour reaction time, the PVDF fibers are fully covered by the ZnO nanoparticles, and the pores between the nanofibers are not blocked by the nanoparticles. XRD analysis of a 1-ZnOPVDF-thick mat was also conducted and the result is shown in Figure 2. In the XRD pattern, all of the characteristic peaks of $\mathrm{ZnO}$ can be observed, verifying that a substantial amount of $\mathrm{ZnO}$ nanoparticles are attached to the PVDF mat.

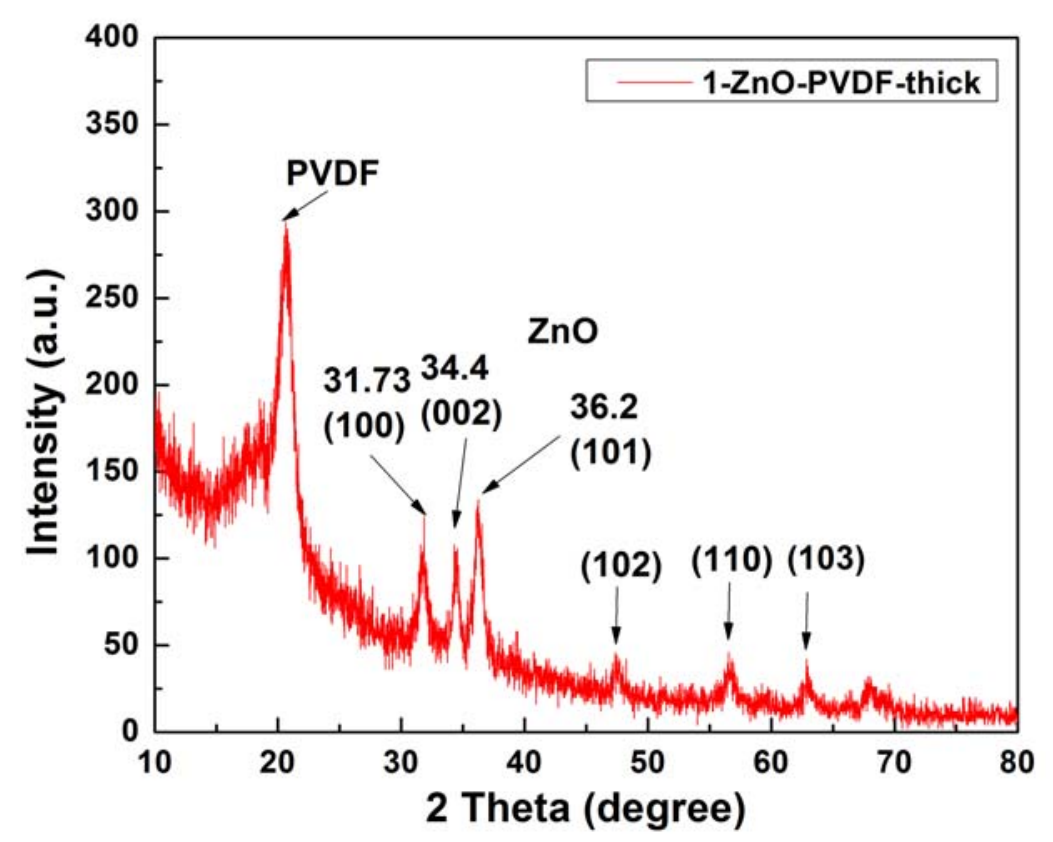

Figure.2 XRD result of 1-ZnO-PVDF-thick mat

\subsection{Anti-bacterial properties of the hybrid mats}


To investigate the effectiveness of this approach in bringing about anti-bacterial behavior and to study the dependence of the antibacterial properties on the amount of $\mathrm{ZnO}$ attached to the PVDF mats, we firstly fabricated thick PVDF nanofibrous mats (about $150 \mu \mathrm{m}$ thick) and attached ZnO nanoparticles onto the mats by suspending the mats in sol-gel solution for different times. The antibacterial properties of Si-PVDF-thick, 0.5-ZnO-PVDF-thick, 1-ZnO-PVDF-thick and 3-ZnOPVDF-thick mats are shown in the optical images in Fig. 3. It can be seen that without $\mathrm{ZnO}$ attachment, the PVDF mat does not show any antibacterial property, and there are plenty of bacteria on the surface of the PVDF mat. With the attachment of ZnO nanoparticles, bacteria are not visible on the surfaces of the hybrid mats, and the inhibition zone becomes larger with the increase of the $\mathrm{ZnO}$ content. The inhibition zone is not uniform on all sides of the samples, which is due to the nonuniform thickness of the electrospun PVDF mats. Nevertheless, the above results indicate that the surface-attached $\mathrm{ZnO}$ nanoparticles are an effective anti-bacterial agent that can prevent growth of bacteria on the ZnO-PVDF mats as well as in the surrounding area.

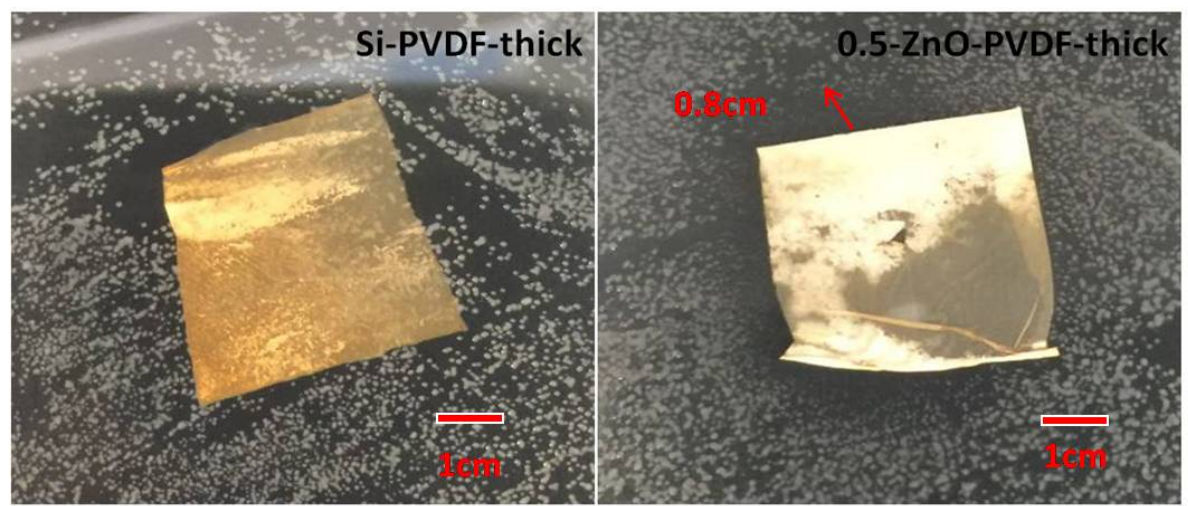

(a)

(b)

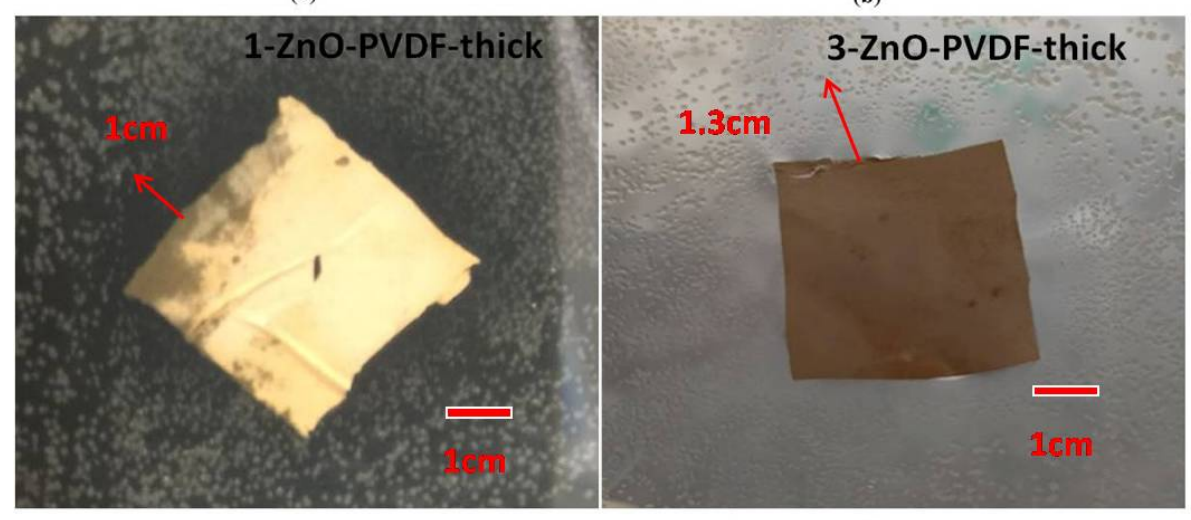

(c)

(d)

Figure.3 Optical images showing the inhibition zone of (a) Si-PVDF-thick, (b) 0.5-ZnO-PVDFthick, (c) 1-ZnO-PVDF-thick, (d) 3-ZnO-PVDF-thick mats. 
In our design, the ZnO-attached PVDF mats are used as the hydrophobic inner layer of the duallayer mat. The hydrophobic layer should be fairly thin in order to let the moisture pass through it quickly and, therefore, thin hybrid mats (ZnO-PVDF, about $5 \mu \mathrm{m}$ thick) were fabricated. In order to prevent folding of the mat and achieve uniform attachment of the $\mathrm{ZnO}$ nanoparticles on the PVDF mat in the sol-gel reaction, the mats collected on aluminum foils were directly suspended in the solgel solution for reaction for $1 \mathrm{hr}$. In the reaction, one side of the mat was fully exposed to the reaction solution, while the other side was in touch with the aluminum foil. The morphologies of both sides of ZnO-PVDF are shown in Figs. 4a and 4b. It can be seen that on the top surface (which was fully exposed to the reaction solution), the $\mathrm{ZnO}$ nanoparticles are uniformly attached to the nanofibers. This is hence denoted as ZnO-PVDF top surface (the side w/ ZnO). By contrast, on the bottom surface (which was in touch with the the aluminum foil) there are almost no $\mathrm{ZnO}$ nanoparticles on the nanofibers, implying that although the thickness of the mat is fairly small, the reaction solution cannot effectively reach the backside of the mat. This side of the ZnO-PVDF mat is hence denoted as $\mathrm{ZnO}-\mathrm{PVDF}$ bottom surface (the side w/o $\mathrm{ZnO}$ ). Owing to their different structures, the properties of the two sides are different.

Figs 4c and 4d are optical images of the inhibition zones after the anti-bacterial test. For both sides, the inhibition zone is not very clear because the amount of $\mathrm{ZnO}$ present in this very thin modified layer is very small. It has been reported that the antibacterial mechanisms of $\mathrm{ZnO}$ include direct contact of $\mathrm{ZnO}$ with cell walls, which results in destruction of bacterial cell integrity, liberation of $\mathrm{Zn}^{2+}$ ions as antimicrobial ions and generation of reactive oxygen species. ${ }^{29-31}$ In this system, since the $\mathrm{ZnO}$ nanoparticles are covalently bonded to the mats, very little $\mathrm{ZnO}$ could diffuse into the culture medium to release $\mathrm{Zn}^{2+}$ ions or generate reactive oxygen species in the culture medium, which is the likely reason for the absence of a clearly visible inhibition zone. Nevertheless, these mats are distinctly different from the neat PVDF mat because no bacterium is visible on either side of the ZnO-PVDF mat.

To further confirm the anti-bacterial property of the ZnO-PVDF mat, the surfaces after the antibacterial test were observed using FESEM to check the presence of bacteria, and the results are shown in Figs. 4e and 4f. It can be seen that there are no bacteria on both sides of the ZnO-PVDF mats. In the experiment, the nanofibrous mats were observed by FESEM without washing after the antibacterial test. The surface of the nanofibers was largely covered with the residues of the culture in the test. Therefore the $\mathrm{ZnO}$ nanoparticles on the nanofibers could not be observed after the test. The above results indicate that the presence of $\mathrm{ZnO}$ nanoparticles on the front surface of the $\mathrm{ZnO}$ - 
PVDF mats can effectively prevent the growth of the bacteria on the front side of the samples. The $\mathrm{ZnO}$ nanoparticles attached to the front surface can also prevent the growth of bacteria on the back side of the mats because the mats are only about $5 \mu \mathrm{m}$ thick. By contrast, the result of our control test shows that the bacteria could grow on neat PVDF mats, confirming that the absence of bacteria on the back side of the thin $\mathrm{ZnO}-\mathrm{PVDF}$ mat is due to the $\mathrm{ZnO}$ and not to the hydrophobic nature of PVDF. The control test result will be presented and discussed in more detail in the section concerning dual-layer mats.

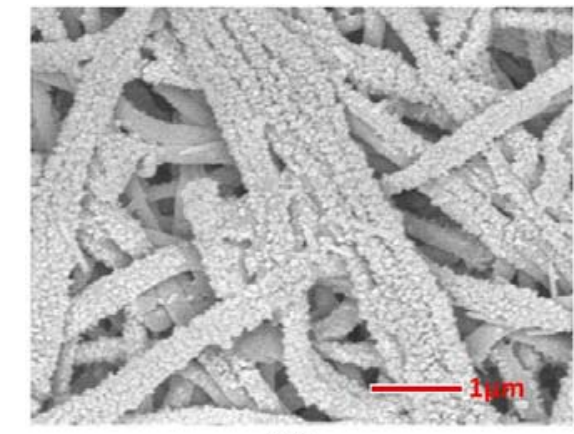

(a)

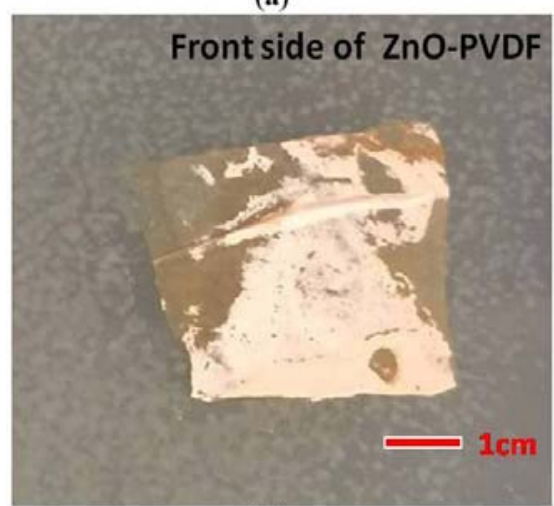

(c)

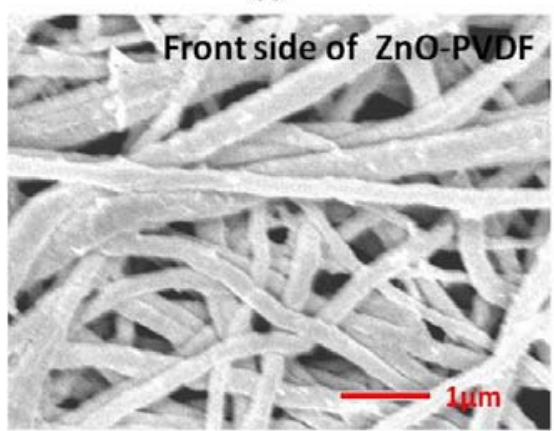

(e)

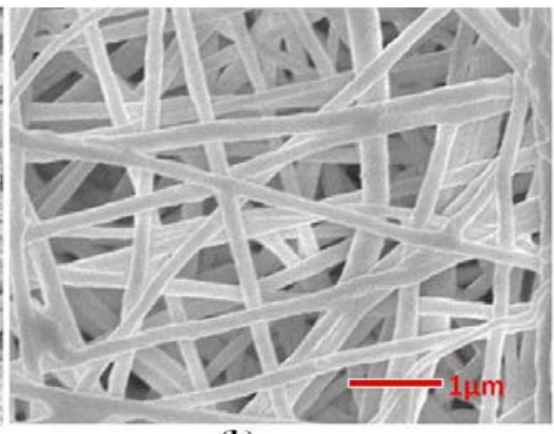

(b)

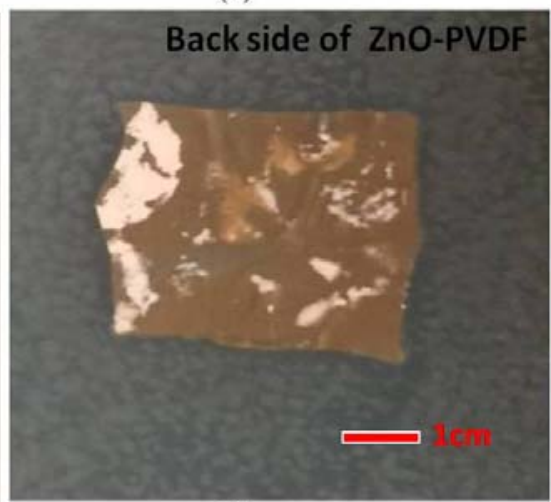

(d)

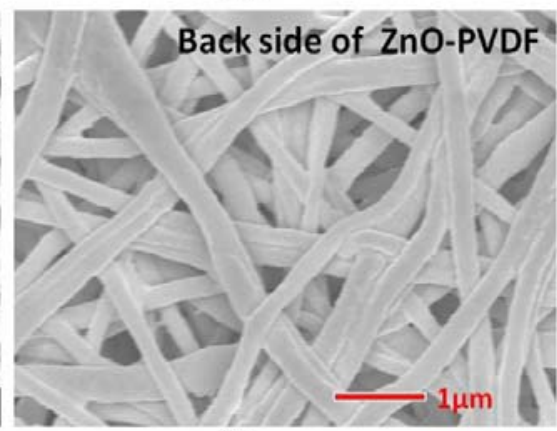

(f)

Figure.4 FESEM micrographs showing the morphologies of (a) front side of ZnO-PVDF, (b) backside of ZnO-PVDF; optical images showing inhibition zone of (c) front side of ZnO-PVDF, (d) backside of ZnO-PVDF; FESEM micrographs showing the morphologies of (e) front side of ZnO-PVDF and (f) backside of ZnO-PVDF after antibacterial test. 


\subsection{Anti-washing property}

Since detachment of $\mathrm{ZnO}$ nanoparticles from the mats would reduce the antibacterial properties of the mats, and the detached nanoparticles may also give rise to potential health risks, a good antiwash property is one of the key criteria for textile applications. In this study, $\mathrm{ZnO}$ is covalently attached onto silane-functionalized PVDF to enhance the anti-wash property. To verify the effectiveness of this method, the $\mathrm{ZnO}$-attached silane-functionalized PVDF (ZnO-PVDF) and ZnOcoated unfunctionalized PVDF (ZnO/PVDF-no silane) mats were washed for both 20 hours and 40 hours and then observed using FESEM. As shown in Fig. 5, the ZnO nanoparticles attached onto the silane-functionalized PVDF can survive well during a 40-hour wash in a detergent solution with continual shaking. However, for the ZnO/PVDF-no silane mat, although $\mathrm{ZnO}$ nanoparticles initially cover the whole surface of the nanofibrous mat, they are washed away after 20-hr of washing and almost disappear completely after 40-hr of washing, presumably due to the lack of strong covalent bonds between the nanoparticles and nanofibers. With severe washing in the detergent solution, the PVDF nanofibers also aggregate together, forming big lumps (Fig. 5c), after the ZnO layer was lost.
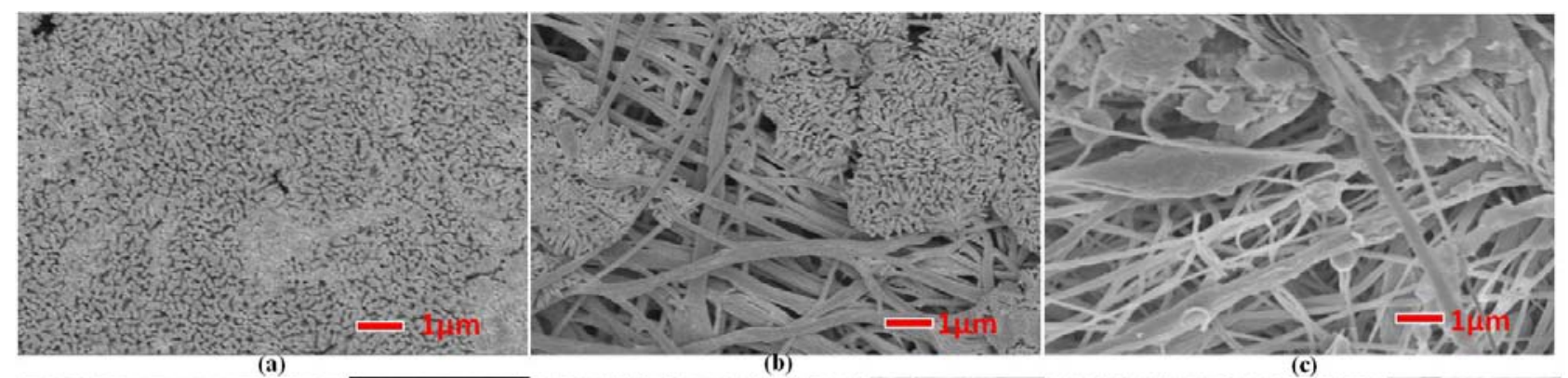

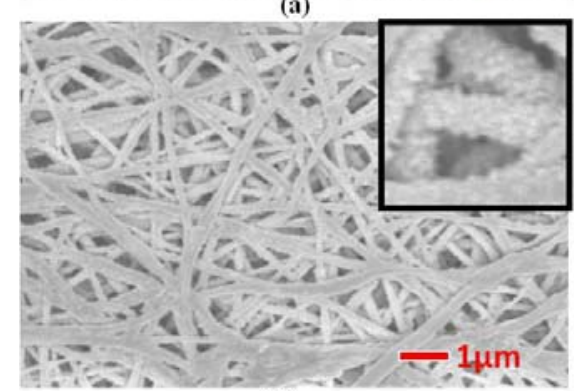

(d)

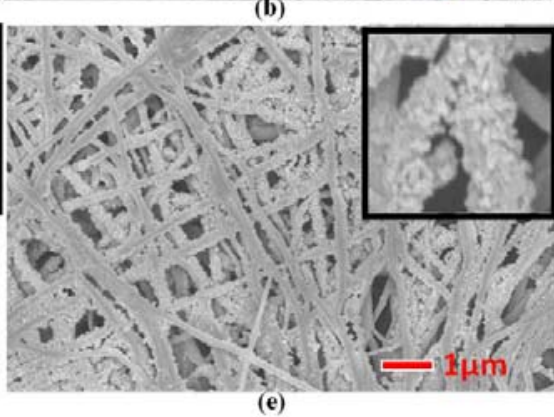

(e)

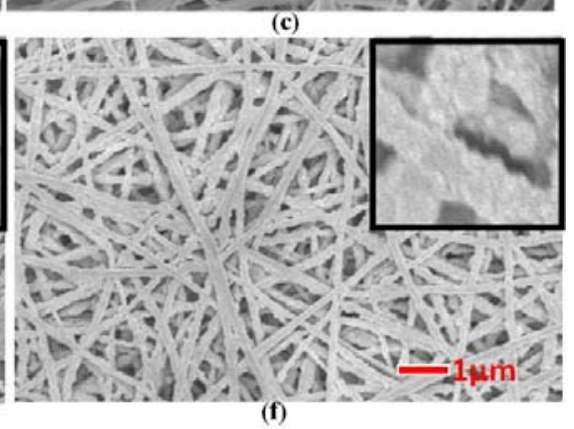

Figure.5 FESEM micrographs showing the morphologies of (a) ZnO/PVDF-no silane, (b) $\mathrm{ZnO} / \mathrm{PVDF}$-no silane after washing for $20 \mathrm{hrs}$, (c) ZnO/PVDF-no silane after washing for $40 \mathrm{hrs,}$ (d) ZnO-PVDF, (e) ZnO-PVDF after washing for $20 \mathrm{hrs,} \mathrm{and} \mathrm{(f)} \mathrm{ZnO-PVDF} \mathrm{after} \mathrm{washing} \mathrm{for} 40$ hrs. 
The good anti-wash property provided by the covalent bonding is further verified by the XRD results shown in Fig. 6. Compared with the results shown in Fig. 2, most characteristic peaks of $\mathrm{ZnO}$, except (101), are not visible due to the very thin nature of the ZnO-PVDF mat. After washing for 20 and 40 hours respectively, the weak (101) peak of $\mathrm{ZnO}$ can still be observed, again suggesting the strong bonding between the nanoparticles and nanofibers. The slight weakening of the X-ray diffraction peaks after washing might be related to crystal disordering at the $\mathrm{ZnO}$ nanoparticle surface caused by washing in detergent and loss of some $\mathrm{ZnO}$ nanoparticles that are not covalently bonded to PVDF. Overall, most $\mathrm{ZnO}$ nanoparticles are retained. Thus, this robust thin hybrid mat was used to fabricate ZnO-PVDF/PAN (the side w/ ZnO next to the PAN layer) and PVDF-ZnO/PAN (the side w/o ZnO next to the PAN layer) dual-layer mats.

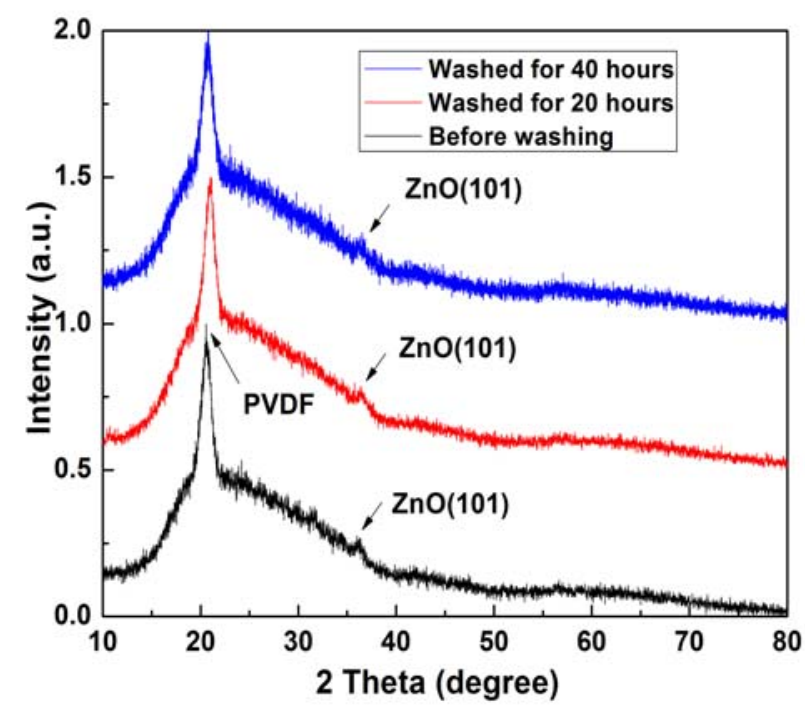

Figure.6 XRD patterns of ZnO-PVDF before washing, after washing for 20 and 40 hrs, respectively.

\subsection{Properties of the dual-layer mats}

To study the moisture transport properties of the dual-layer nanofibrous mats, the wettability of the mats and their components should be investigated first. In our design, an inner hydrophobic ZnOPVDF layer (the layer next to skin) is combined with a hydrophilic PAN outer layer to induce the push-pull effect to drive the moisture out from the skin and prevent wet fabric from sticking to the skin. With this design, the inner hydrophobic layer has to be very thin to allow water to pass quickly through it, ensuring good water transport through the dual-layer mats. At the same time, the hydrophilic PAN layer should be relatively thick in order to pull the moisture out efficiently. Considering that water evaporation at the surface of the PAN layer takes time, with a very thin 
PAN layer that has a very small volume for holding water, the push-pull effect would easily cause the water content of the PAN layer to reach a high level, diminishing the push-pull effect. Thus, a thin ZnO-PVDF layer ( $\sim \mu \mathrm{m}$ thick) and a thick PAN layer ( 100 $\mu \mathrm{m}$ thick) are used as the inner and outer layers of the dual-layer mats, respectively.

To study the wettability of the mats, the time-dependent apparent contact angles of the samples were tested. In this case, since the electrospun non-woven mats have numerous inter-penetrating micron-sized pores, even if the material is hydrophobic a very small amount of water can penetrate into the mats slowly due to gravity. In order to distinguish the contact angle measured on such porous mats from that measured on a solid surface, we use the term "apparent contact angle". Usually, the time-dependent apparent contact angle is determined by both water spreading on the testing surface and penetrating into the pores of the mats. The images in Figure 7a show the changes of the apparent contact angles with time, and the test results are summarized in Figure $7 \mathrm{~b}$. The neat PVDF mat is very hydrophobic. During the test, although a small amount of water can penetrate into the PVDF mats very slowly due to gravity and hence the volume of the droplet decreases slightly, the droplet cannot spread on the surface of the PVDF mat at all (Fig. 7a(i)) owing to the high hydrophobicity of PVDF and the lotus effect. Hence the apparent contact angle does not change with the droplet volume reduction. The apparent contact angle of the PVDF mat is almost a constant in the test period of ten minutes. By contrast, PAN is hydrophilic and hence the water could both spread on the surface and penetrate into the mats, causing it to disappear in less than 3 seconds (Fig. 7a (ii)).

As already mentioned, the thin ZnO-PVDF mat has two distinctly different surfaces, one consists of PVDF nanofibers fully covered with $\mathrm{ZnO}$ nanoparticles ( $\mathrm{ZnO}$ side) while the other consists of neat PVDF nanofibers (PVDF side). The ZnO side is more hydrophilic, and the water droplet could penetrate into the mat in 100 seconds (Fig. 7a(iii). When a PAN layer is added at the bottom of the ZnO-PVDF layer with the $\mathrm{ZnO}$ side on top to form a ZnO-PVDF/PAN dual-layer mat, the water could penetrate into the mat faster due to the push-pull effect induced by the difference in hydrophilicity of the two layers. However, although the volume of the water droplet on the surface reduces quickly for the dual-layer mat (Fig. 7a(iv)), the time dependent behaviour of the apparent contact angle of the dual layer mat is almost identical to that of the ZnO-PVDF single-layer mat with the $\mathrm{ZnO}$ side on top (Fig. 7b). 
In contrast, the phenomenon observed from the ZnO-PVDF single-layer mat with the PVDF side on top is close to that of the neat PVDF film, i.e., the droplet penetrates into the mats very slowly (in nearly 7 minutes, Fig. 7a(v)). In addition, when a PAN layer is added to the bottom of the $\mathrm{ZnO}$ PVDF layer with the PVDF surface on top to form a PVDF-ZnO/PAN dual-layer mat, the time dependent behaviour of the apparent contact angle of this dual layer mat is quite different from that of the ZnO-PVDF single-layer mat with the PVDF side on top; the water could penetrate into the PVDF-ZnO/PAN mat faster, in only 140 seconds. Thus after combination with a hydrophilic PAN mat, the hydrophilicity difference between the two layers also induces a push-pull effect, although the resultant water transport in PVDF-ZnO/PAN is slower than that in ZnO-PVDF/PAN (Fig. 7b).
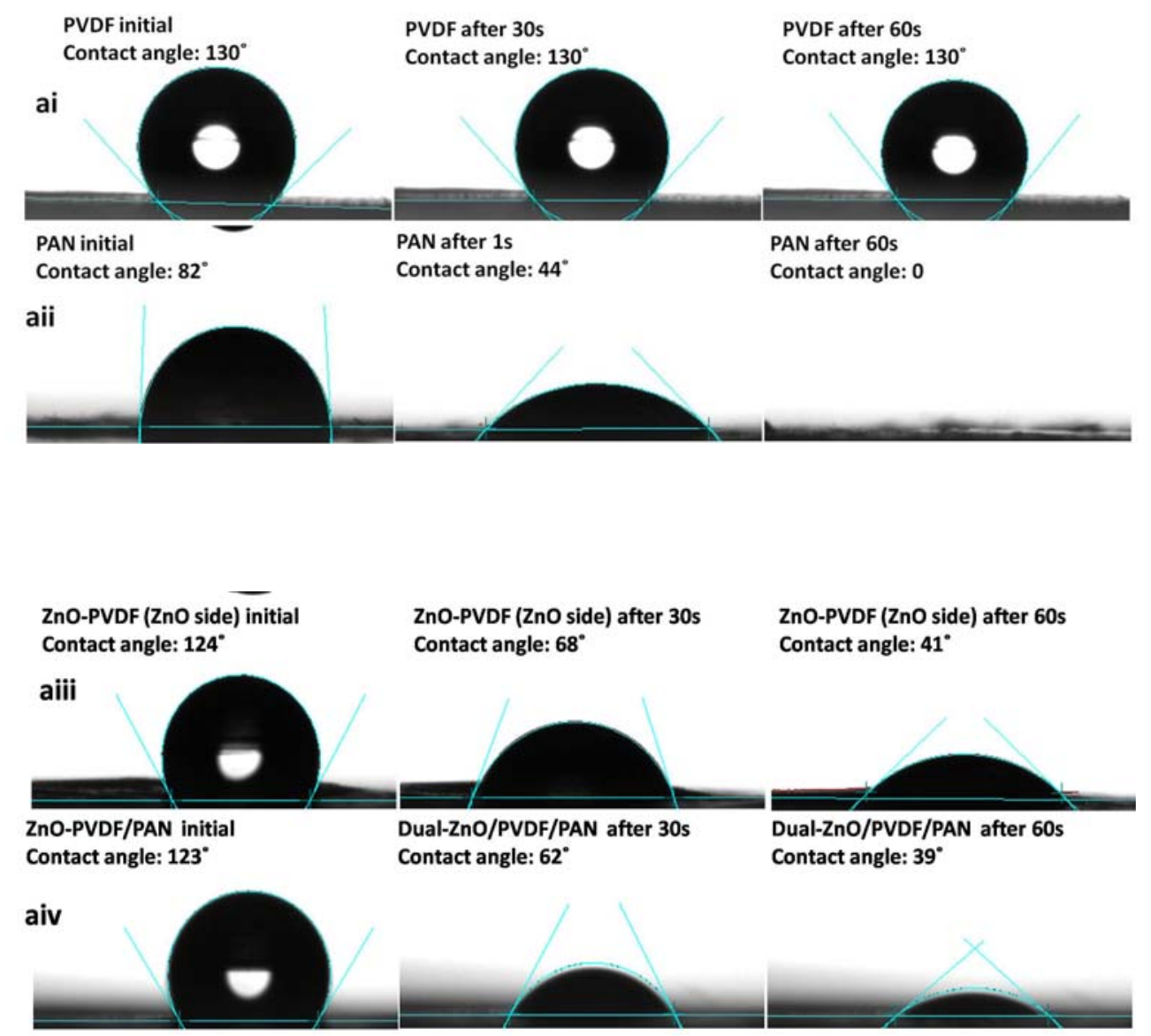

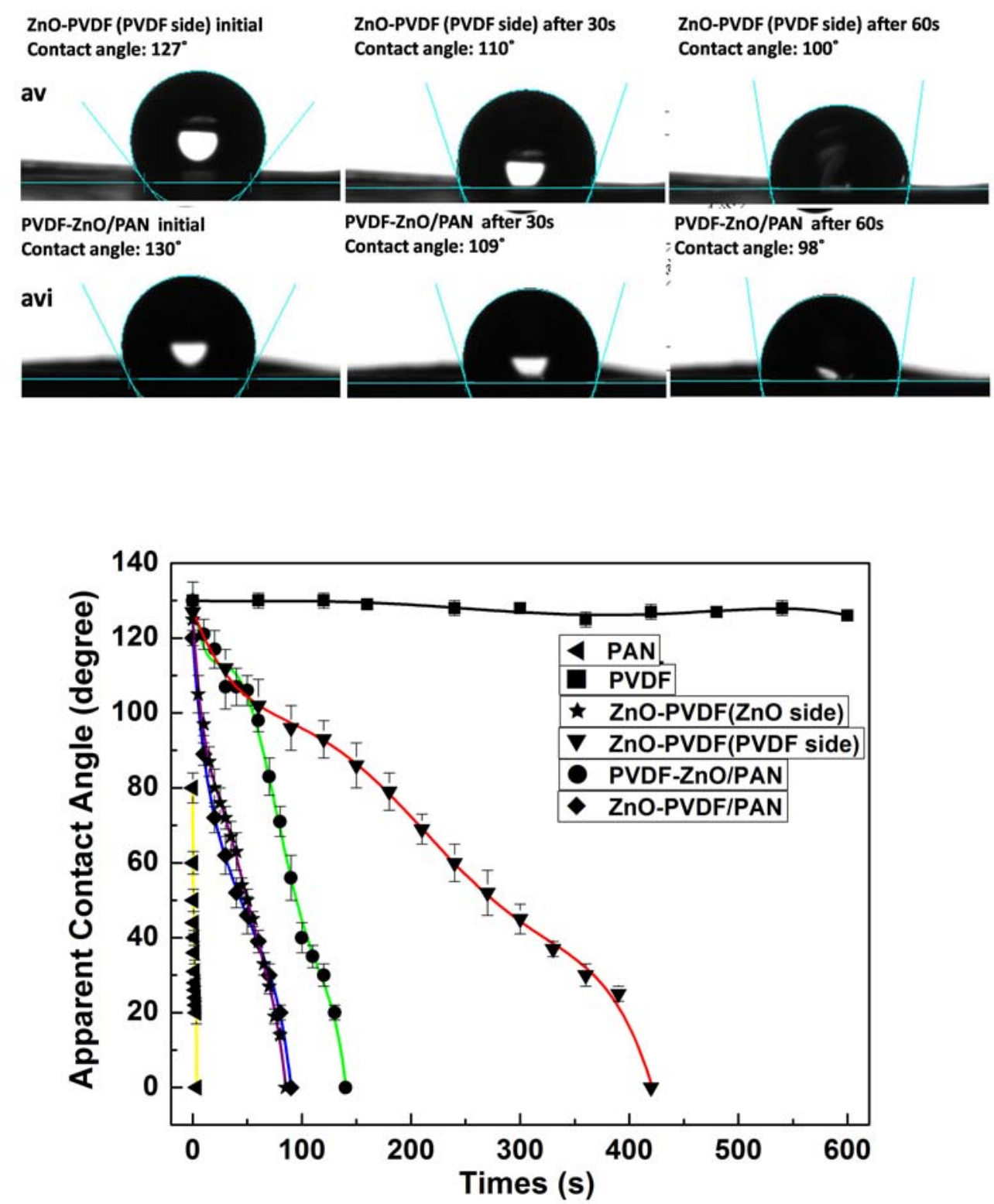

(b)

Figure.7 The images (a) and curves (b) showing time-dependent contact angle of $\mathrm{ZnO}$ side and PVDF side of ZnO-PVDF, PVDF, PAN, PVDF-ZnO/PAN and ZnO-PVDF/PAN.

As explained above, since the ZnO-PVDF electrospun mat has interpenetrating pores, a small amount of water can penetrate into the mat due to gravity, and in the ZnO-PVDF/PAN dual-layer mat water will be pulled into the PAN layer due to the hydrophilicity difference between the two layers. This enhances the moisture transport property of the dual-layer mat. However, the apparent contact angle measurements cannot adequately characterize such water transport behaviour because although the apparent contact angles of the ZnO-PVDF single-layer and ZnO-PVDF/PAN duallayer mats are similar, their water transport mechanisms are different. Thus, the MMT tester is needed to further study the water transport behaviour of the dual-layer mats. 


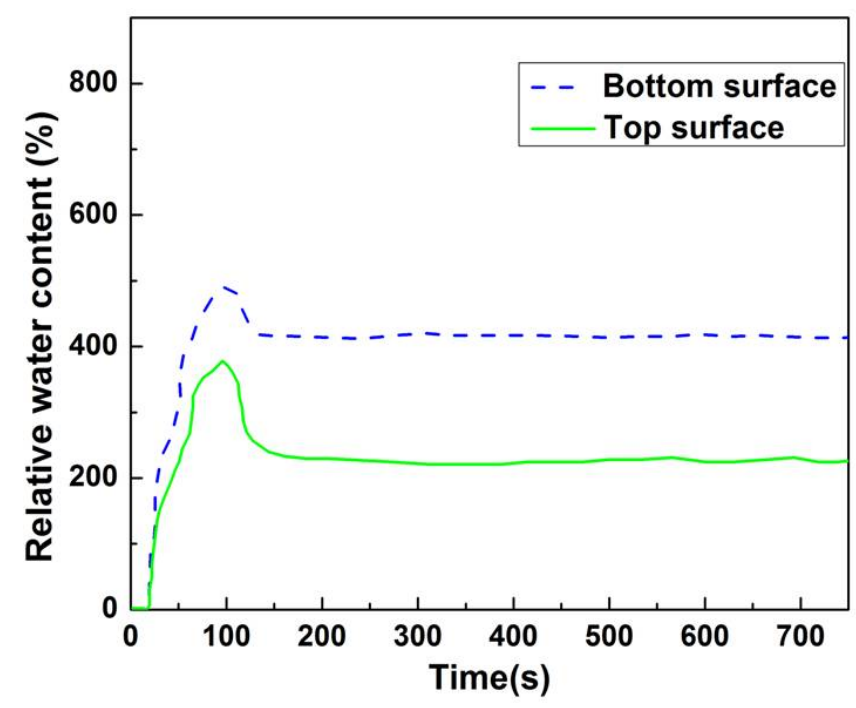

(a)

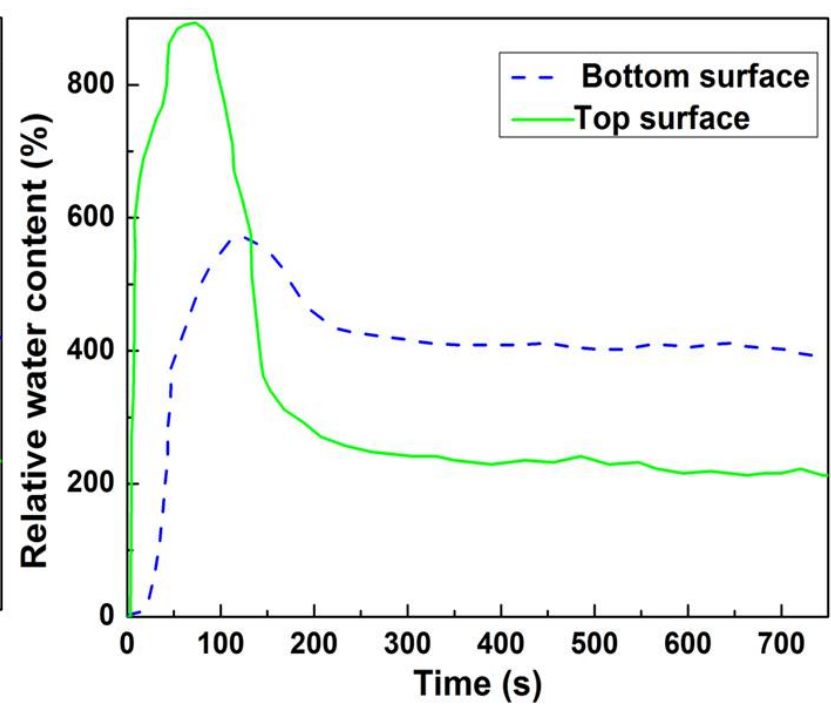

(b)

Figure 8. MMT results for (a) ZnO-PVDF/PAN and (b) PVDF-ZnO/PAN mats. PAN is the bottom layer.

The MMT results for the ZnO-PVDF/PAN and PVDF-ZnO/PAN dual-layer mats are shown in Fig. 8. The solid green line indicates the water content of the top surface ( $\mathrm{ZnO}$ or PVDF) where the water drops are sprayed initially, while the dashed blue line indicates the water content of the bottom surface (PAN). For the ZnO-PVDF/PAN mat, since the top surface has reasonably good wettability owing to the attachment of ZnO nanoparticles on PVDF, water could wet the surface quickly. The bottom layer is made of PAN nanofibers, which are more hydrophilic than the top layer. Water can hence be pulled from the top to the bottom layer quickly. Therefore, the water content of the bottom surface is much higher, demonstrating the push-pull effect. For the PVDF$\mathrm{ZnO} / \mathrm{PAN}$ mat, since the top surface is mainly made of PVDF which is more hydrophobic than $\mathrm{ZnO}$, water could not penetrate into the mats very quickly due to the poor wettability and the lotus effect. As a result, the water content of the top surface is fairly high at the beginning. After around 80 seconds, water penetrates into the mats and the water content of the top layer starts to reduce, until the water content of the top layer is much lower than that of the bottom layer. The MMT results are consistent with the results of time-dependent contact angle measurements, indicating that the ZnO-PVDF/PAN mats have better moisture transport properties than the PVDF-ZnO/PAN mats. Thus, for dual-layer mats, antibacterial tests were only conducted for ZnO-PVDF/PAN mats.

The antibacterial properties of the ZnO-PVDF surface of the ZnO-PVDF/PAN dual-layer mats were tested with neat PVDF and PAN mats as controls. The inhibition zone of the dual-layer mats could not be observed clearly, as found for the corresponding single-layer ZnO-PVDF mats (Figs. 
4c and 4d). Thus, to verify the antibacterial properties of the dual-layer mats, they were observed under FESEM after the antibacterial tests. As shown in Figs. 9a and 9b, the surfaces of neat PVDF and PAN mats are completely covered by bacteria after the antibacterial tests, showing that they do not have any antibacterial property. By contrast, there are no bacteria on both sides of the ZnOPVDF/PAN mat (Figs. 9c and 9d). The observations indicate that the ZnO-PVDF surface of the dual-layer mats exhibits reasonably good antibacterial properties.

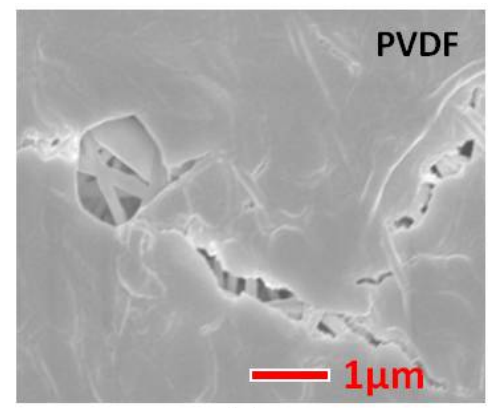

(a)

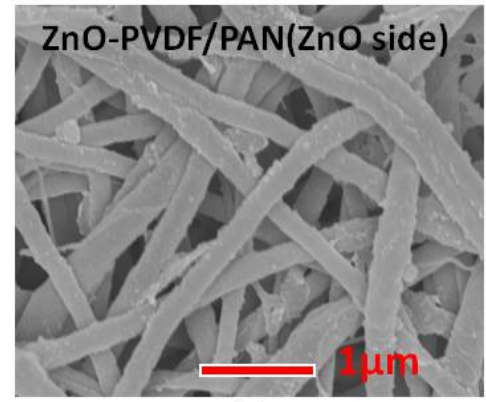

(c)

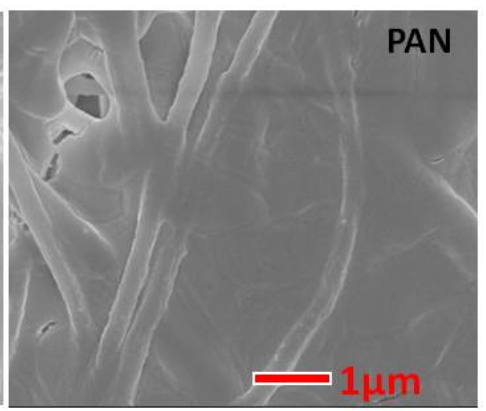

(b)

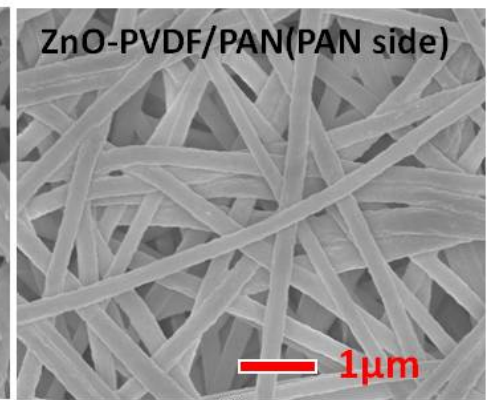

(d)

Figure 9. FESEM micrographs showing the morphologies of (a) PVDF, (b) PAN, (c) ZnOPVDF/PAN (PVDF side), (d) ZnO-PVDF/PAN (PAN side) mats after antibacterial tests.

\section{Conclusions}

In this paper, dual-layer nanofibrous mats composed of a thick hydrophilic PAN nanofibrous layer and a hydrophobic PVDF nanofibrous layer with covalently attached ZnO nanoparticles are designed and fabricated. The antibacterial property of the dual-layer mats rendered by the surfacelocated $\mathrm{ZnO}$ nanoparticles is verified, and it is found that the hybrid mats with covalently bonded $\mathrm{ZnO}$ nanoparticles have good anti-wash properties. The dual layer mats also show good moisture transport properties and therefore could provide a drier environment between the skin and textile, which can enhance the wear comfort and reduce the growth of bacteria. 


\section{References}

1 Uttam, D. Active sportswear fabrics. International Journal of IT, Engineering and Applied Sciences Research 2, 34-40 (2013).

2 Fan, J. \& Cheng, X.-Y. Heat and moisture transfer with sorption and phase change through clothing assemblies Part I: Experimental investigation. Text. Res. J. 75, 99-105 (2005).

3 Troynikov, O. \& Wardiningsih, W. Moisture management properties of wool/polyester and wool/bamboo knitted fabrics for the sportswear base layer. Text. Res. J. 81, 621-631 (2011). Zeng, C., Wang, H., Zhou, H. \& Lin, T. Directional Water Transport Fabrics with Durable Ultra-High One-Way Transport Capacity. Advanced Materials Interfaces 3, n/a-n/a (2016). Sivamani, R. K., Goodman, J., Gitis, N. V. \& Maibach, H. I. Coefficient of friction: tribological studies in man-an overview. Skin Research and Technology 9, 227-234 (2003). Hendriks, C. \& Franklin, S. Influence of surface roughness, material and climate conditions on the friction of human skin. Tribology Letters 37, 361-373 (2010).

7 Wilhelm, K.-P., Elsner, P., Berardesca, E. \& Maibach, H. I. Bioengineering of the skin: skin imaging \& analysis. (CRC Press, 2006).

8 Neves, S. F., Campos, J. B. L. M. \& Mayor, T. S. Effects of clothing and fibres properties on the heat and mass transport, for different body heat/sweat releases. Applied Thermal Engineering 117, 109-121 (2017).

9 Mondal, S., Reddy, V., Sarkar, A., Aravindakshan, P. \& Ghatak, A. Effect of surface modification on frictional properties of polyester fabric. Tribology International 97, 38-48 (2016).

10 Zaman, M., Liu, H., Xiao, H., Chibante, F. \& Ni, Y. Hydrophilic modification of polyester fabric by applying nanocrystalline cellulose containing surface finish. Carbohydr. Polym. 91, 560-567 (2013).

11 Salas, C., Genzer, J., Lucia, L. A., Hubbe, M. A. \& Rojas, O. J. Water-wettable polypropylene fibers by facile surface treatment based on soy proteins. ACS Appl. Mater. Interfaces 5, 6541-6548 (2013).

12 Srinivasan, J., Ramakrishnan, G., Mukhopadhyay, S. \& Manoharan, S. A study of knitted fabrics from polyester microdenier fibres. Journal of the Textile Institute 98, 31-35 (2007).

13 Süpüren, G., Oglakcioglu, N., Ozdil, N. \& Marmarali, A. Moisture management and thermal absorptivity properties of double-face knitted fabrics. Text. Res. J., 81(13) 13201330 (2011).

14 Dong, Y. et al. Tailoring surface hydrophilicity of porous electrospun nanofibers to enhance capillary and push-pull effects for moisture wicking. ACS Appl. Mater. Interfaces 6, 1408714095 (2014).

15 Dastjerdi, R. \& Montazer, M. A review on the application of inorganic nano-structured materials in the modification of textiles: focus on anti-microbial properties. Colloids and Surfaces B: Biointerfaces 79, 5-18 (2010).

16 Chen, L., Bromberg, L., Hatton, T. A. \& Rutledge, G. C. Electrospun cellulose acetate fibers containing chlorhexidine as a bactericide. Polymer 49, 1266-1275 (2008).

17 Ravindra, S., Mohan, Y. M., Reddy, N. N. \& Raju, K. M. Fabrication of antibacterial cotton fibres loaded with silver nanoparticles via “Green Approach”. Colloids Surf., A 367, 31-40 (2010).

18 Perelshtein, I., Lipovsky, A., Perkas, N., Tzanov, T. \& Gedanken, A. Sonochemical codeposition of antibacterial nanoparticles and dyes on textiles. Beilstein journal of nanotechnology 7, 1-8 (2016).

19 Doumbia, A. S., Vezin, H., Ferreira, M., Campagne, C. \& Devaux, E. Studies of polylactide/zinc oxide nanocomposites: influence of surface treatment on zinc oxide antibacterial activities in textile nanocomposites. J. Appl. Polym. Sci. 132 (2015). 
20 Hatamie, A. et al. Zinc Oxide Nanostructure-Modified Textile and Its Application to Biosensing, Photocatalysis, and as Antibacterial Material. Langmuir 31, 10913-10921 (2015).

21 Raghupathi, K. R., Koodali, R. T. \& Manna, A. C. Size-dependent bacterial growth inhibition and mechanism of antibacterial activity of zinc oxide nanoparticles. Langmuir 27, 4020-4028 (2011).

22 Koga, H., Kitaoka, T. \& Wariishi, H. In situ synthesis of silver nanoparticles on zinc oxide whiskers incorporated in a paper matrix for antibacterial applications. J. Mater. Chem. 19, 2135-2140 (2009).

23 Schwartz, V. B. et al. Antibacterial surface coatings from zinc oxide nanoparticles embedded in poly ( $\mathrm{n}$ - isopropylacrylamide) hydrogel surface layers. Advanced Functional Materials 22, 2376-2386 (2012).

24 Wang, Y.-W. et al. Superior antibacterial activity of zinc oxide/graphene oxide composites originating from high zinc concentration localized around bacteria. ACS Appl. Mater. Interfaces 6, 2791-2798 (2014).

25 Gupta, A., Kim, B. C., Edwards, E., Brantley, C. \& Ruffin, P. Covalent functionalization of zinc oxide nanowires for high sensitivity p -nitrophenol detection in biological systems. Materials Science \& Engineering B 177, 1583-1588 (2012).

26 Zhou, R. et al. Chemically cross-linked ultrathin electrospun poly(vinylidene fluoride-cohexafluoropropylene) nanofibrous mats as ionic liquid host in electrochromic devices. Polymer 55, 1520-1526 (2014).

27 Olena Taratula et al. Binding Studies of Molecular Linkers to ZnO and MgZnO Nanotip Films. Journal of Physical Chemistry B 110, 6506-6515 (2006).

28 Gupta, A. et al. Surface Chemistry of ZnO Nanowires for Nanosensor Applications. Journal of Nanotechnology in Engineering and Medicine 2, 011010-011014, doi:10.1115/1.4003306 (2011).

29 Dutta, R. K., Nenavathu, B. P., Gangishetty, M. K. \& Reddy, A. V. R. Studies on antibacterial activity of $\mathrm{ZnO}$ nanoparticles by ROS induced lipid peroxidation. Colloids \& Surfaces B Biointerfaces 94, 143 (2012).

30 Raghupathi, K. R., Koodali, R. T. \& Manna, A. C. Size-dependent bacterial growth inhibition and mechanism of antibacterial activity of zinc oxide nanoparticles. Langmuir the Acs Journal of Surfaces \& Colloids 27, 4020-4028 (2011).

31 Applerot, G. et al. Enhanced Antibacterial Activity of Nanocrystalline ZnO Due to Increased ROS - Mediated Cell Injury. Advanced Functional Materials 19, 842-852 (2009). 